\title{
Voltage Dependent Anion Channel Is Redistributed during Japanese Encephalitis Virus Infection of Insect Cells
}

\author{
Chanida Fongsaran, ${ }^{1}$ Narumon Phaonakrop, ${ }^{2}$ Sittiruk Roytrakul, ${ }^{2}$ Chutima Thepparit, ${ }^{1}$ \\ Atichat Kuadkitkan, ${ }^{1}$ and Duncan R. Smith ${ }^{1,3}$ \\ ${ }^{1}$ Institute of Molecular Biosciences, Mahidol University, Salaya Campus, 25/25 Phuttamonthol Sai 4 Road, Salaya, \\ Nakhon Pathom 73170, Thailand \\ ${ }^{2}$ Proteomics Research Laboratory, Genome Institute, National Science and Technology Development Agency, 113 Thailand Science Park, \\ Phahonyothin Road, Khlong Nueng, Khlong Luang, Pathum Thani 12120, Thailand \\ ${ }^{3}$ Center for Emerging and Neglected Infectious Diseases, Mahidol University, 25/25 Phuttamonthon 4 Road, Salaya, \\ Nakhon Pathom 73170, Thailand
}

Correspondence should be addressed to Duncan R. Smith; duncan_r_smith@hotmail.com

Received 9 April 2014; Accepted 25 June 2014; Published 10 July 2014

Academic Editor: Mehmet Yakup Arica

Copyright (C) 2014 Chanida Fongsaran et al. This is an open access article distributed under the Creative Commons Attribution License, which permits unrestricted use, distribution, and reproduction in any medium, provided the original work is properly cited.

\begin{abstract}
Despite the availability of an effective vaccine, Japanese encephalitis remains a significant cause of morbidity and mortality in many parts of Asia. Japanese encephalitis is caused by the Japanese encephalitis virus (JEV), a mosquito transmitted flavivirus. Many of the details of the virus replication cycle in mosquito cells remain unknown. This study sought to determine whether GRP78, a wellcharacterized flavivirus E protein interacting protein, interacted with JEV E protein in insect cells, and whether this interaction was mediated at the cell surface. GRP78 was shown to interact with JEV E protein by coimmunoprecipitation, and was additionally shown to interact with voltage dependent anion protein (VDAC) through the same methodology. Antibody inhibition experiments showed that neither GRP78 nor VDAC played a role in JEV internalization to insect cells. Interestingly, VDAC was shown to be significantly relocalized in response to JEV infection, and significant levels of colocalization between VDAC and GRP78 and VDAC and ribosomal L28 protein were seen in JEV infected but not uninfected cells. This is the first report of relocalization of VDAC in response to JEV infection and suggests that this may be a part of the JEV replication strategy in insect cells.
\end{abstract}

\section{Introduction}

Japanese encephalitis virus (JEV) is a mosquito transmitted virus of the genus Flavivirus, family Flaviviridae [1]. The genome of JEV is a $5^{\prime}$ capped, single-stranded, positive-sense RNA molecule of approximately $11 \mathrm{~kb}[2]$ with a single open reading frame that encodes three structural proteins and seven nonstructural proteins required for viral replication [3]. JEV is distributed in much of Asia and Northern Australia, with most cases of human infection occurring in China, India, and Southeast Asia $[4,5]$. JEV is maintained in a natural enzootic cycle between water birds and mosquitoes, although in rural areas pigs are an important amplifying reservoir $[4,6]$. Culex tritaeniorhynchus mosquitoes are the primary transmission vector in India and Southeast Asian countries [7], and vertical transmission of JEV has been reported for this and other mosquito species $[4,6]$. Humans can be infected when bitten by an infected mosquito, but humans are a dead end host for virus transmission due to low levels of viraemia [4]. While the majority of cases of human infection with JEV are believed to be asymptomatic [8], an estimated 30,000 to 50,000 cases of Japanese encephalitis (JE) occur annually, resulting in some 10,000 to 15,000 deaths $[4,9,10]$.

JEV enters into mammalian cells by at least two different methods of endocytosis. The virus is apparently internalized through a clathrin independent mechanism in cells of a neuronal origin [11, 12], while cells of a nonneuronal origin apparently internalize the virus through clathrin dependent mechanisms [11, 13, 14]. A number of proteins including 
hsp70 [15, 16], vimentin [17], the low density lipoprotein receptor [18], and CD14 and CD4 [19] have been implicated in playing a role as an initial JEV binding or receptor protein mediating the attachment and internalization of JEV to a variety of mammalian cells.

Significantly less is known about the events mediating JEV entry and replication in insect cells. Susceptible mosquitoes become infected after a blood meal from a viraemic animal [4], although the basis of mosquito susceptibility remains unknown. It is likely however that as with other flavivirus/susceptible host mosquito systems, vectorial competence is determined by up to three genetically determined barriers, namely, the midgut barrier, a midgut escape barrier, and a salivary escape barrier [20]. Studies have suggested that JEV enters into insect cells by endocytosis [21] and hsc70 has been proposed as a putative receptor molecule [22]. Both protein elements and glycosaminoglycans have been implicated in the entry of JEV into insect cells [23].

GRP78 is a multifunctional chaperone protein that has been shown to interact with dengue virus $\mathrm{E}$ protein in a number of studies [24-26]. GRP78 is predominantly localized to the endoplasmic reticulum (ER) where it regulates the activation of the unfolded protein response (UPR) in response to stress conditions [27-29]. However, cell surface GRP78 expression is well documented [24, 30-33], and GRP78 has been implicated as a virus receptor for dengue $[24,34]$ and coxsackievirus A9 [35]. This study sought to initially determine if there was an interaction between GRP78 or the GRP78 interacting protein VDAC (voltage dependent anion channel) and JEV E protein in insect cells and to determine whether the interaction had any relevance to virus internalization.

\section{Material and Methods}

2.1. Cell and Virus. The Aedes albopictus cell line C6/36 was cultured at $28^{\circ} \mathrm{C}$ in minimum essential medium (MEM, Gibco Invitrogen, Carlsbad, CA) supplemented with $10 \%$ heat-inactivated fetal bovine serum (FBS, Gibco Invitrogen) and 100 units/mL of penicillin and $100 \mu \mathrm{g} / \mathrm{mL}$ of streptomycin (PAA Laboratories, Linz, Austria). LLC-MK 2 cells (rhesus monkey kidney cell line) were cultured in Dulbecco's modified eagle's medium (DMEM, Gibco Invitrogen) supplemented with $5 \%$ FBS with the same antibiotics at $37^{\circ} \mathrm{C}$ in humidified incubator with $5 \% \mathrm{CO}_{2}$. The Japanese encephalitis virus strain Beijing-1 (accession number L48961) was propagated in C6/36 cells as described elsewhere [23] and virus stock was prepared by partial purification by centrifugation to remove cell debris and supplementation with 20\% FBS before storage at $-80^{\circ} \mathrm{C}$. Virus titer was determined by standard plaque assay using LLC-MK $\mathrm{MK}_{2}$ cells as described previously [36].

2.2. Virus Infection. C6/36 cells were grown under standard conditions until they reached confluence after which they were incubated with JEV in MEM media at the indicated multiplicity of infection (m.o.i.) for $2 \mathrm{hrs,} \mathrm{after} \mathrm{which} \mathrm{the}$ cells were washed with PBS to remove uninternalized virus before being incubated again under standard conditions for the times indicated.

2.3. Coimmunoprecipitation Assay. C6/36 cells were grown to $80 \%$ confluence in $100 \mathrm{~mm}^{2}$ tissue culture plates and either not infected or infected with JEV at MOI of 10 at $28^{\circ} \mathrm{C}$ for $2 \mathrm{~h}$. Cells were cultured under standard conditions for 3 days after infection after which the cells were collected by centrifugation, washed with PBS, and lysed using Co-IP lysis buffer (25 mM Tris- $\mathrm{HCl} \mathrm{pH}$ 7.4, $150 \mathrm{mM} \mathrm{NaCl}, 1 \mathrm{mM}$ EDTA, 1\% NP40, 5\% glycerol, $1 \mathrm{mM}$ PMSF, and $1 \mathrm{mM} \mathrm{Na}_{3} \mathrm{VO}_{4}$ ) followed by incubation on ice for $5 \mathrm{~min}$ before centrifugation at $16000 \times \mathrm{g}$ for $5 \mathrm{~min}$. The protein concentration was determined by the Bradford method [37]. To preclear the lysates, $1 \mathrm{mg}$ equivalent of lysates was diluted 2:3 and incubated with Protein G Sepharose 4 Fast Flow (GE Healthcare, Buckinghamshire, $\mathrm{UK}$ ) at $4^{\circ} \mathrm{C}$ on a rotator for $2 \mathrm{hrs}$. Subsequently, $100 \mu \mathrm{L}$ of precleared lysates were incubated with $1 \mu \mathrm{g}$ of a rabbit monoclonal anti-VDAC antibody or a 1:10 dilution of monoclonal antibody $\mathrm{HB} 112$ with gentle rocking overnight at $4^{\circ} \mathrm{C}$. To precipitate protein complexes, $20 \mu \mathrm{L}$ of protein $\mathrm{G}$ was added to the solutions, which were incubated with gentle rocking at $4^{\circ} \mathrm{C}$ for $4 \mathrm{hrs}$. The mixtures were collected by centrifugation at $6000 \times \mathrm{g}$ for $5 \mathrm{~min}$ and the supernatants were discarded. The pellets were washed twice with CoIP-lysis buffer and resuspended in $30 \mu \mathrm{L}$ of $3 x$ SDS sample loading buffer and the proteins were heated to $100^{\circ} \mathrm{C}$ for $5 \mathrm{~min}$ followed by centrifugation at $14000 \times \mathrm{g}$ for 2 min twice before applying the samples to $12 \%$ SDS-polyacrylamide gels for subsequent western blot analysis.

2.4. Western Blotting. Coimmunoprecipitated proteins were separated by electrophoresis through 12\% SDS-polyacrylamide gels and subsequently transferred to solid support (nitrocellulose membranes) using the Trans-Blot electrophoretic transfer cell (Bio-Rad Laboratories, Richmond, CA). The membranes were blocked with $5 \%$ skimmed milk in TBS at room temperature for $2 \mathrm{~h}$. For western blot analysis, the membranes were incubated with a $1: 1000$ dilution of a goat polyclonal anti-GRP78 antibody (SC-1050; Santa Cruz Biotechnology Inc., Santa Cruz, CA) followed by a 1:2000 dilution of a HRP-conjugated rabbit anti-goat IgG antibody (31402, Pierce, Thermo Fisher Scientific Inc., Rockford, IL) at room temperature for $2 \mathrm{~h}$. Final signal was detected by using the ECL Plus Western Blotting analysis kit (GE Healthcare).

2.5. Antibody Mediated Infection Inhibition Assay. C6/36 cells were grown in six-well plates and the cells were then incubated with $20 \mu \mathrm{g}$ of a rabbit polyclonal anti-VDAC antibody (sc-98708; Santa Cruz Biotechnology Inc.) or $20 \mu \mathrm{g}$ of a goat polyclonal anti-GRP78 antibody (sc-1050; Santa Cruz Biotechnology Inc.) or $20 \mu \mathrm{g}$ of a combination of each antibody or $20 \mu \mathrm{g}$ of a mouse monoclonal anti- $\alpha \mathrm{V} / \beta 5$ integrin antibody (sc-13588; Santa Cruz Biotechnology Inc.) or no antibody at $28^{\circ} \mathrm{C}$ for 1 . After incubation, the cells were incubated with JEV at MOI of 10 for $2 \mathrm{~h}$ at $28^{\circ} \mathrm{C}$. The cells were washed with $1 x$ PBS and treated with acid glycine, 


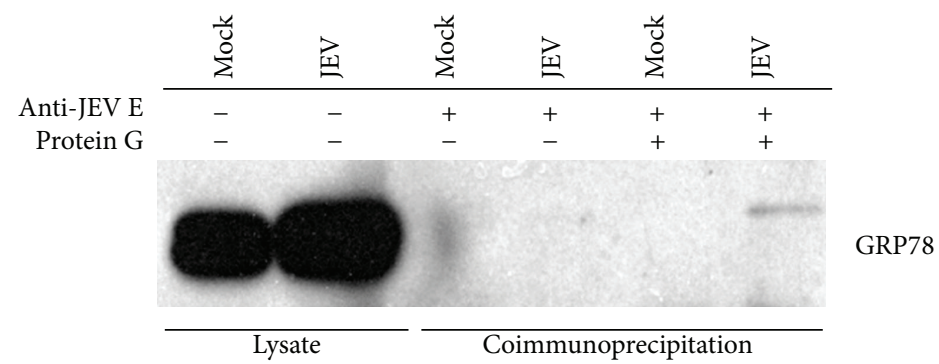

(a)

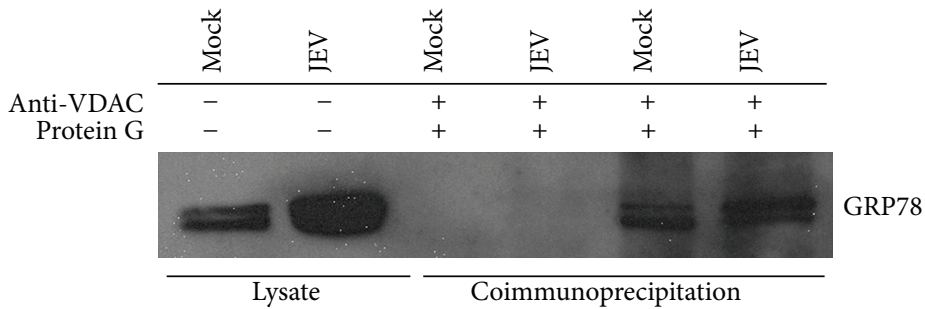

(b)

FIGURE 1: Coimmunoprecipitation analysis of GRP78 and JEV E protein and GRP78 and VDAC. C6/36 cells were mock and or infected with JEV for V days, after which cells were lysed and either E protein (a) or VDAC (b) immunoprecipitated with an appropriate antibody. Coimmunoprecipitation of GRP78 was determined by electrophoresis of the precipitated proteins and transfered to solid matrix support after which membrane was probed with an antibody against GRP78.

$\mathrm{pH}$ 3, for 1 min to remove uninternalized virus [38]. The cells were washed with 1x PBS and fresh growth medium was added. Cells were collected at 8 hours after infection and analyzed by flow cytometry as described previously [19]. Each sample was analyzed in duplicate and the experiment was done independently in triplicate.

2.6. Confocal Microscopy and Image Analysis. For intracellular colocalization analysis cells were grown to $60 \%$ confluence on glass coverslips before being infected with JEV at m.o.i. 10 for $2 \mathrm{hrs}$ and further cultured under standard protocol. At 24 hrs postinfection cells were fixed with ice-cold absolute methanol for $20 \mathrm{~min}$, washed with PBS, and permeabilized by incubation with $0.3 \%$ Triton X-100 in PBS for 10 min before blocking with 5\% BSA in PBS for 1 hr. Cells were subsequently incubated with three different primary antibodies simultaneously overnight at $4^{\circ} \mathrm{C}$ after which cells were washed two times with PBS and incubated with three appropriate secondary antibodies for $1 \mathrm{hr}$ at room temperature before examination under an Olympus FluoView 1000 microscope equipped with Olympus FluoView software version 1.6.

Primary antibodies used were a 1:10 dilution of a pan-specific antiflavivirus $\mathrm{E}$ protein monoclonal antibody (HB112), a 1:50 dilution of rabbit polyclonal anti-VDAC antibody (sc-98708; Santa Cruz Biotechnology Inc.), a 1:50 dilution of goat polyclonal anti-GRP78 antibody (sc-1050; Santa Cruz Biotechnology Inc.), and a 1:50 dilution of goat polyclonal antiribosomal protein L-28 antibody (sc-14151; Santa Cruz Biotechnology Inc.). Secondary antibodies used were a 1:100 dilution of an Alexa 488-conjugated donkey antimouse IgG antibody (A21202; Molecular Probes, Thermo
Fisher Scientific Inc.), a 1:100 dilution of an Alexa 647conjugated donkey anti-rabbit IgG antibody (A31573; Molecular Probes), a 1:100 dilution of an Alexa 568-conjugated donkey anti-goat IgG antibody (A11057; Molecular Probes), a 1:200 dilution of an Alexa 594-conjugated chicken antimouse IgG antibody (A21201; Molecular Probes), a 1:50 dilution of a FITC-conjugated donkey anti-rabbit IgG antibody (sc-2090; Santa Cruz Biotechnology Inc.), and a 1:100 dilution of a Cy5-conjugated rabbit anti-goat IgG antibody (81-1616; Invitrogen, Thermo Fisher Scientific Inc.).

Image analysis was undertaken as described previously [39] using the ImageJ analysis program [40] and the PSC colocalization plugin [41]. At least 20 cells were analyzed for each condition. Results are presented in terms of the Pearson correlation coefficients, with statistical analysis of significance between datasets undertaken by a paired sample test using SPSS (SPSS Inc.).

\section{Results}

To determine whether GRP78 interacts with JEV E protein in insect cells, C6/36 cells were mock infected or infected with JEV and at 3 days after infection JEV E protein was pulled down from the cell lysates using an antiflavivirus $\mathrm{E}$ protein monoclonal antibody. The pulled-down proteins were separated by electrophoresis and transferred to solid matrix and the membrane subsequently probed with an anti-GRP78 polyclonal antibody. Results (Figure 1(a)) showed that GRP78 was pulled down in complex with JEV E protein.

Studies in mammalian cells have shown that GRP78 interacts with VDAC at the cell surface [42-44], and so it was determined whether there was an interaction between 


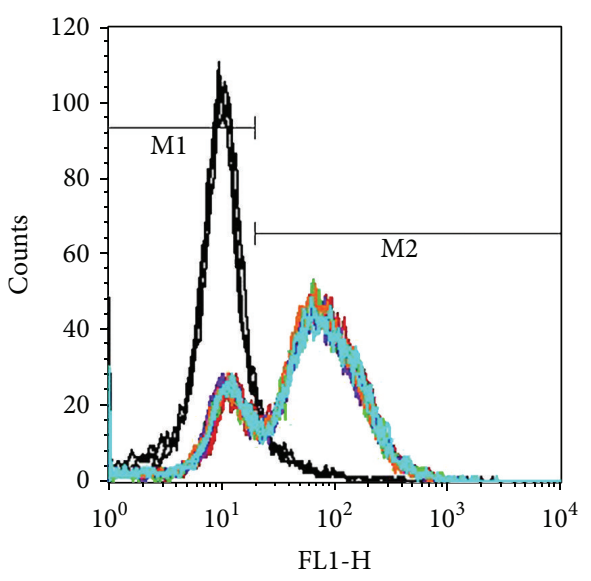

(a)

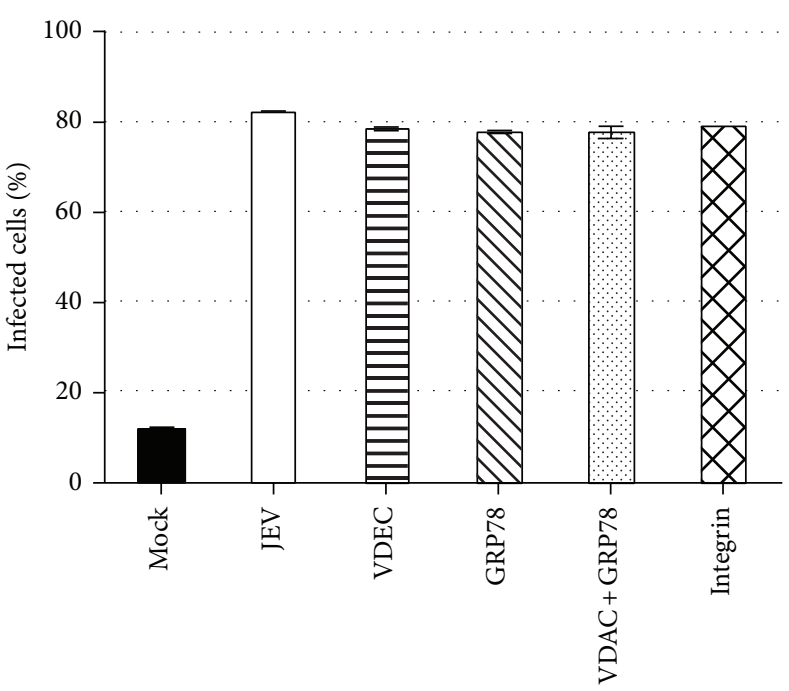

(b)

FIGURE 2: Antibody inhibition analysis of JEV entry to insect cells. C6/36 cells were pretreated with antibodies against GRP78, VDAC, VDAC, and GRP78 and integrin $\alpha \mathrm{V} / \beta 5$ or no antibody before infection with JEV at MOI of 10 . After 8 hours the percent infection was determined by flow cytometry. Representative raw data profiles (a) and tabular analysis (b) from three independent experiments in duplicate are shown.

GRP78 and VDAC in insect cells. C6/36 cells were either mock infected or infected with JEV, and VDAC precipitated with an anti-VDAC antibody and the precipitated proteins were again separated by electrophoresis, transferred to solid support, and probed with an anti-GRP78 antibody. Results (Figure 1(b)) showed that GRP78 was coprecipitated with VDAC. The interaction between GRP78 and VDAC was seen in both infected and uninfected cells, as would be expected, while the interaction between GRP78 and JEV E protein was confined to infected cells. Repeated pull-down experiments failed to demonstrate an interaction between JEV E protein and VDAC (data not shown).

We next determined whether either of these two proteins (VDAC or GRP78) was acting as JEV receptor proteins through antibody inhibition experiments. C6/36 cells were either mock infected or infected with JEV or infected with JEV after incubation with antibodies directed against VDAC, GRP78, GRP78, and VDAC combined or $\alpha \mathrm{V} / \beta 5$ integrin as an irrelevant control antibody. Results (Figure 2) showed no inhibition of virus entry under any of the conditions tested.

We next determined whether there was intracellular colocalization between VDAC and GRP78 in both uninfected and JEV infected C6/36 cells. C6/36 cells were either mock infected or infected and at 24 hours after infection they were permeabilized and examined for localization under a confocal microscope. A triple staining technique was employed, with primary antibodies directed against JEV E protein, VDAC, and GRP78. As can be seen in Figure 3, there was significantly increased colocalization between GRP78 and VDAC in infected cells as compared to uninfected cells. For easier visualization, the signals for GRP78 and VDAC only are shown in Figure 4. Analysis of colocalization showed that there was a significant increase in colocalization between GRP78 and VDAC in infected cells (Pearson correlation coefficient $0.63 \pm 0.069$ ) as compared to mock infected cells (Pearson correlation coefficient $0.47 \pm 0.067 ; P<0.01$ ).

GRP78 is predominantly localized to the ER, while VDAC is predominantly localized to the outer membrane of the mitochondria. The increased colocalization of GRP78 and VDAC in JEV infected cells would suggest a relocalization of VDAC to the ER in response to infection. To determine if this was the case, C6/36 cells were again either mock infected or infected with JEV and at 24 hours after infection they were examined for the localization of JEV E protein, ribosomal protein L28, and VDAC. As can be seen in Figure 5, a significant increase in colocalization of VDAC and ribosomal protein L28 was observed in JEV infected cells (Pearson correlation coefficient $0.58 \pm 0.045$ ) as compared to mock infected cells (Pearson correlation coefficient $0.41 \pm 0.091$; $P<0.01)$. For easier visualization the signals for VDAC and ribosomal L28 protein only are shown in Figure 6.

\section{Discussion}

GRP78, which is also known as BiP (binding immunoglobulin protein), is a multifunctional protein that has been implicated in a wide range of cellular processes [45]. GRP78 is a member of the heat shock protein 70 (HSP70) family of chaperones and has been best characterized as a central mediator of the endoplasmic reticulum unfolded protein response [2729]. In unstressed conditions, GRP78 binds to and sequesters three UPR regulatory proteins, PERK, ATF6, and IreI, and upon the induction of ER stress by a number of mechanisms including viral infection, PERK, ATF6, and IreI are released from GRP78 leading to the transcriptional regulation of a number of stress response genes [27-29]. In addition to functions in the ER, GRP78 has been identified as a cell surface expressed protein in a number of cell types [24, 30-33], 

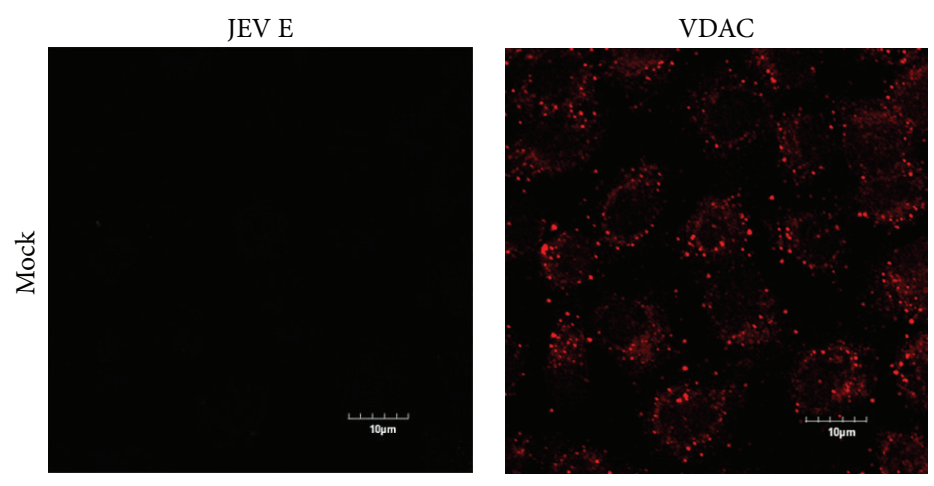

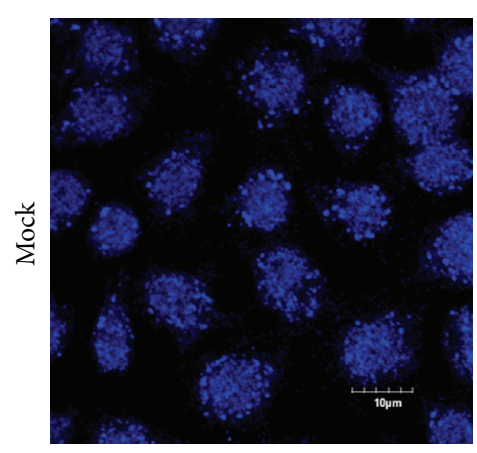

GRP78

JEV E
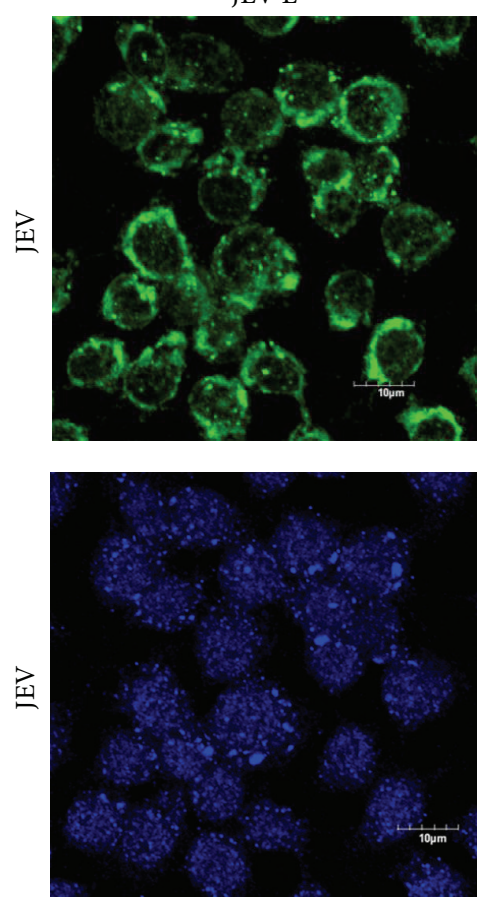

GRP78

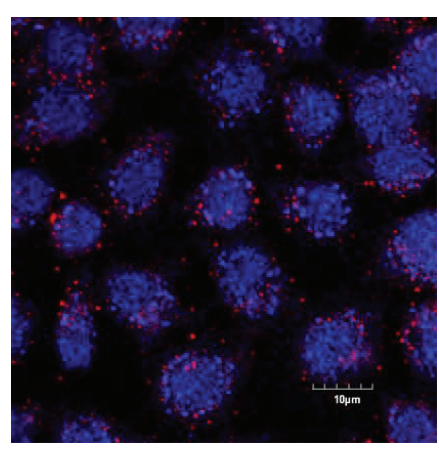

Merge

VDAC
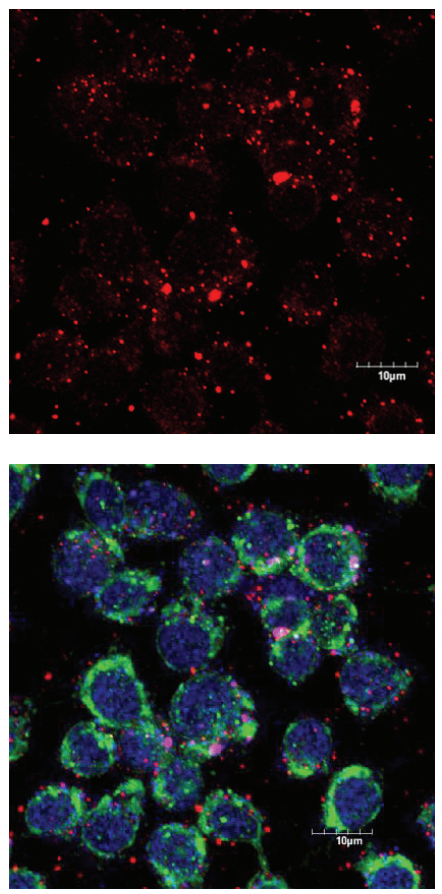

Merge
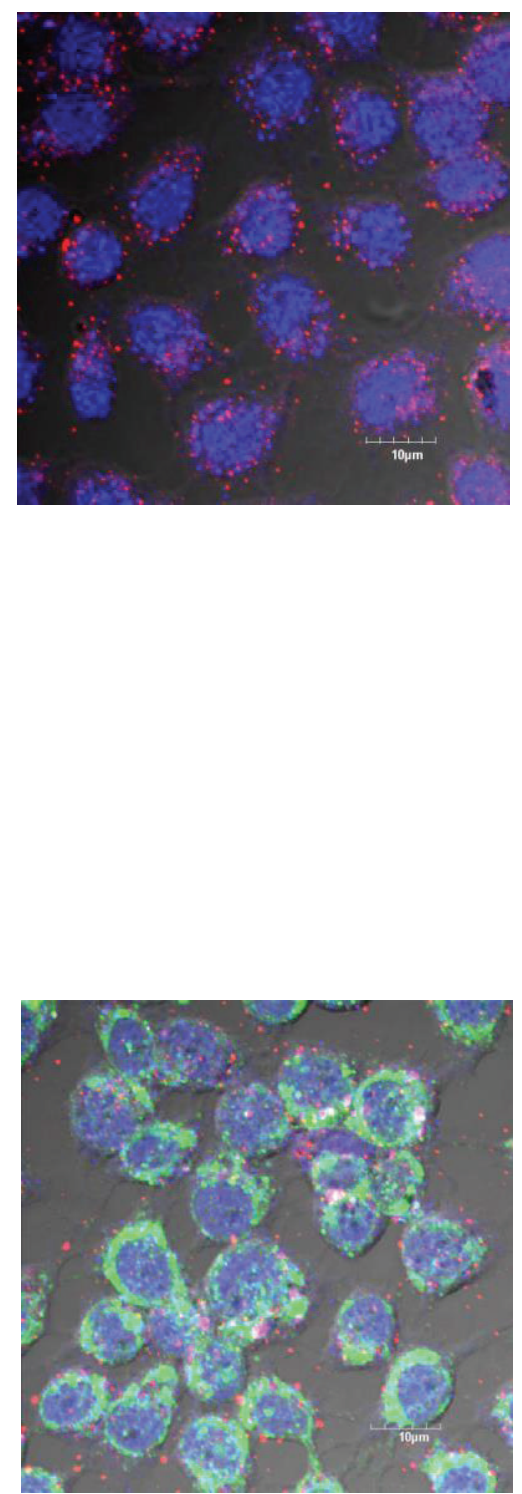

FIGURE 3: Location of JEV E protein, VDAC, and GRP78 in JEV infected C6/36 cells. C6/36 cells grown on glass cover slips were mock infected or infected with JEV and examined for location of JEV E protein (green), VDAC (red), and GRP78 (blue) using an Olympus FluoView 1000 confocal microscope. Representative individual and merged images are shown, and one panel has bright field added. 

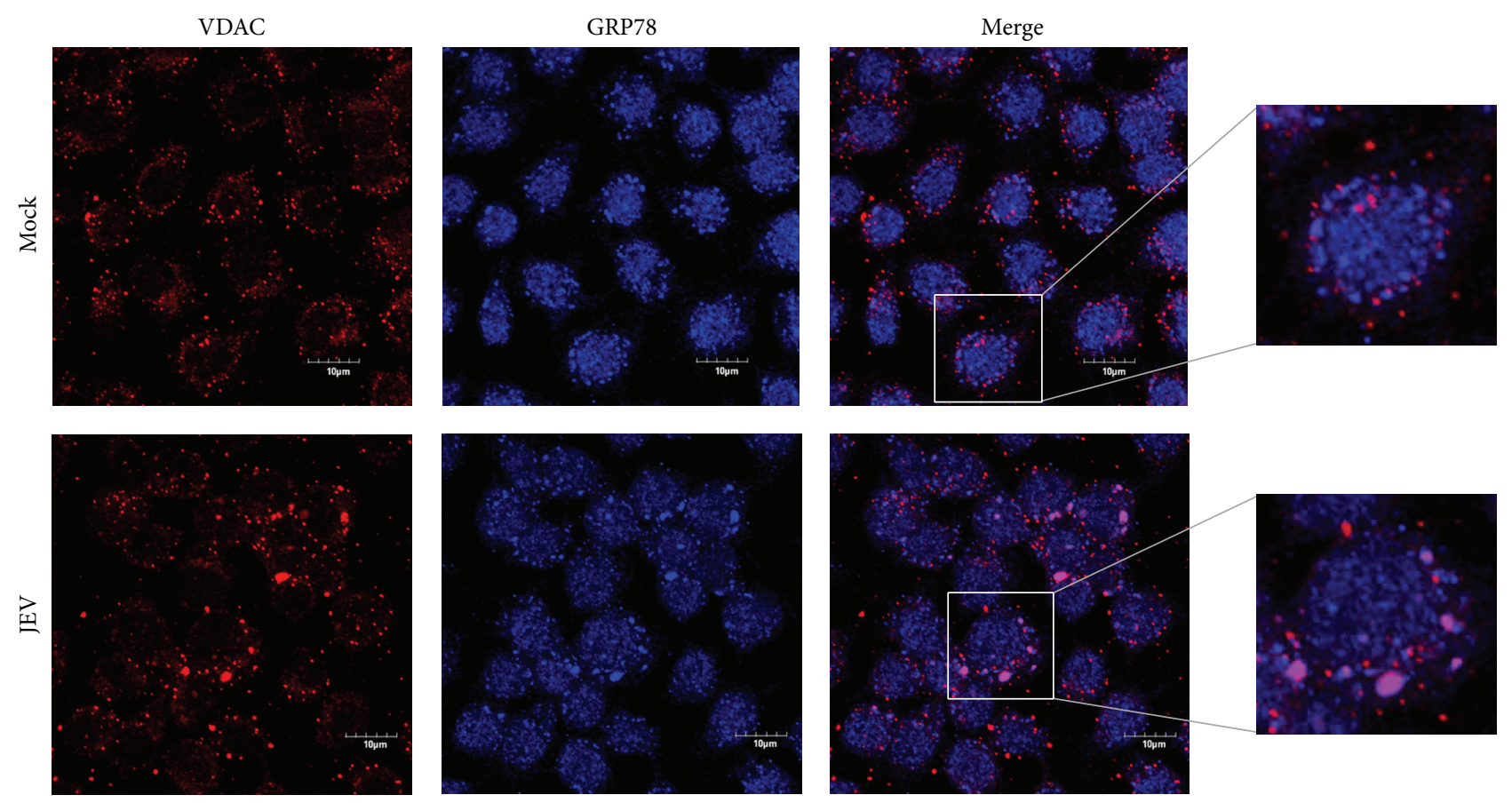

FIGURE 4: Location of VDAC and GRP78 in JEV infected C6/36 cells. Signal of VDAC and GRP78 extracted from Figure 3 for improved visualization purposes with enlargement.

where it acts as a receptor for a number of ligands [43]. GRP78 has been found in association with a number of proteins on the cell surface, including VDAC, the major histocompatibility complex class 1 (MHC-1) and the teratocarcinoma-derived growth factor 1 (Cripto 1) [43].

It was shown that dengue enters into HepG2 cells via GRP78 [24], and independent confirmation showed that downregulation of GRP78 significantly reduced virus entry [34]. Interactions between GRP78 and dengue E protein have been proposed to occur at several steps of the cell cycle, and both cell surface and intracellular interactions between GRP78 and dengue E protein have been documented [24-26].

This study showed that like DENV E protein, JEV E protein is also able to interact with GRP78; however, while GRP78 has been identified as a possible receptor protein for DENV, we found no evidence that GRP78 was able to mediate internalization of JEV to insect cells, either alone or together with VDAC. GRP78 was also excluded as a receptor protein for JEV in a recent study investigating possible JEV receptor proteins expressed by microglial cells [19].

VDAC, also known as mitochondrial porin, is located in the outer mitochondrial membrane (OMM) and maintains the permeability of the outer mitochondrial membrane [46]. VDAC serves to mediate interactions between mitochondria and the rest of the cell by regulating the transport of ions, ATP, and other metabolites [47]. In addition to other functions, VDAC is believed to play a major role in the regulation and execution of apoptosis through its interactions with members of the Bcl2 family of proteins [48], which mediate release of apoptotic proteins present in the inner membranal space.
VDAC has been shown to be associated with GRP78 at the cell surface [42-44], but as shown here, VDAC neither alone nor in combination with GRP78 appears to mediate the internalization of JEV.

As noted, VDAC is known to be predominantly localized in the OMM and additionally at the cell surface. We have shown that in response to JEV infection VDAC shows a significant increase in colocalization with the ER resident GRP78. The fact that VDAC relocalizes to colocalize with GRP78 in the ER, rather than GRP78 relocalizing to colocalize with VDAC at the mitochondria, was shown both by the physical alteration of VDAC localization in response to JEV infection and the fact that JEV infection increased colocalization between ribosomal L28 protein and VDAC.

Several studies have previously observed the close localization of VDAC with the ER. Some studies have suggested that this arises from mitochondria physically being in close association with the ER, which may be coupled with relocalization of VDAC within the mitochondria to form VDAC enriched microdomains that are physically in close contact with the ER [49], while other studies have suggested that VDAC itself is physically located within the ER [50]. Either way, our study shows that in response to JEV infection, VDAC is redistributed to be in close contact with the ER in insect cells. As noted, VDAC mediates the release of ions and a number of metabolites including ATP [47], and a recent study with DENV NS3 has suggested that ATP levels may be a critical component in balancing the unwinding and annealing activities of the helicase portion of NS3 [51]. As such, the relocalization of VDAC would suggest that this is an 

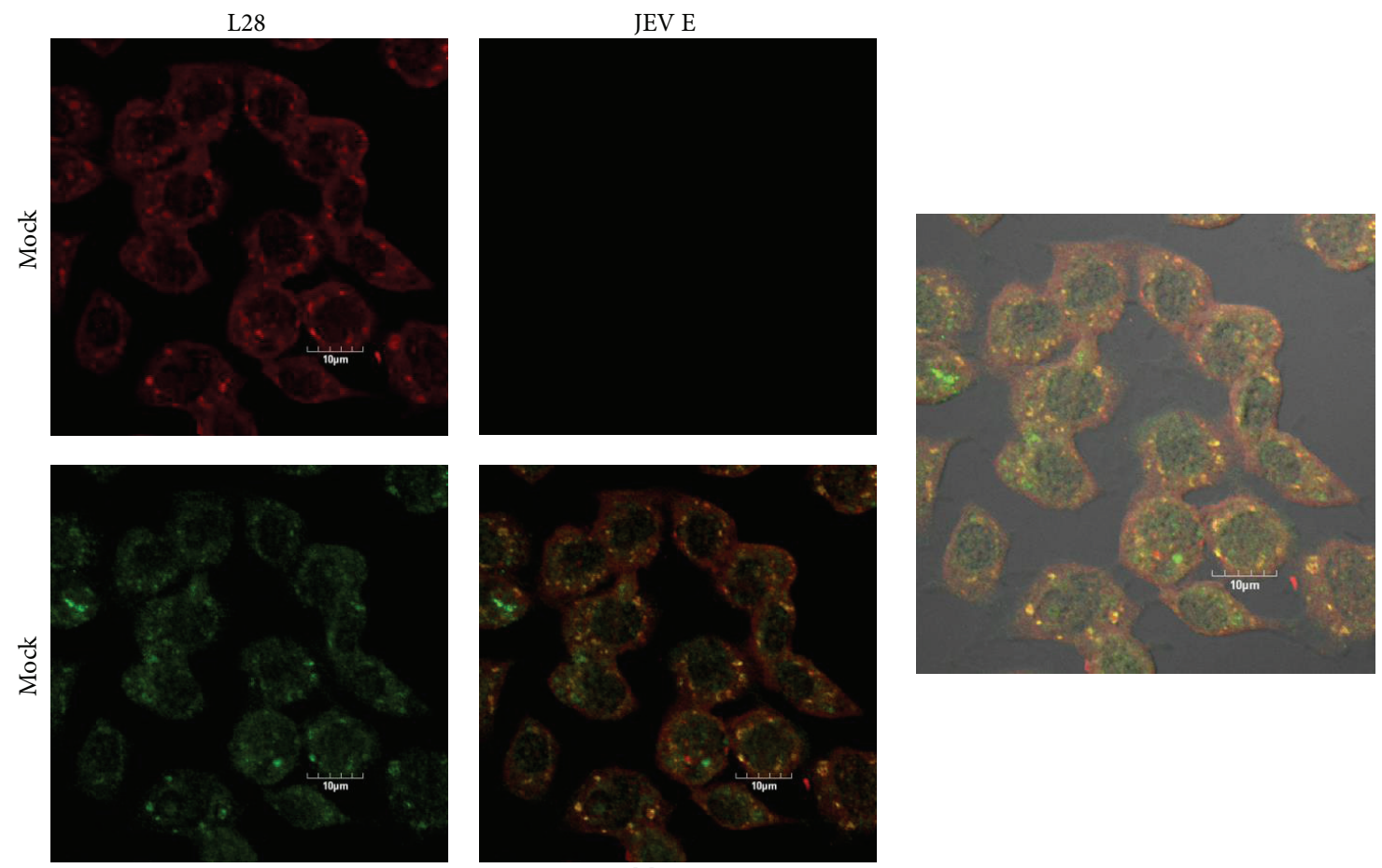

VDAC

Merge
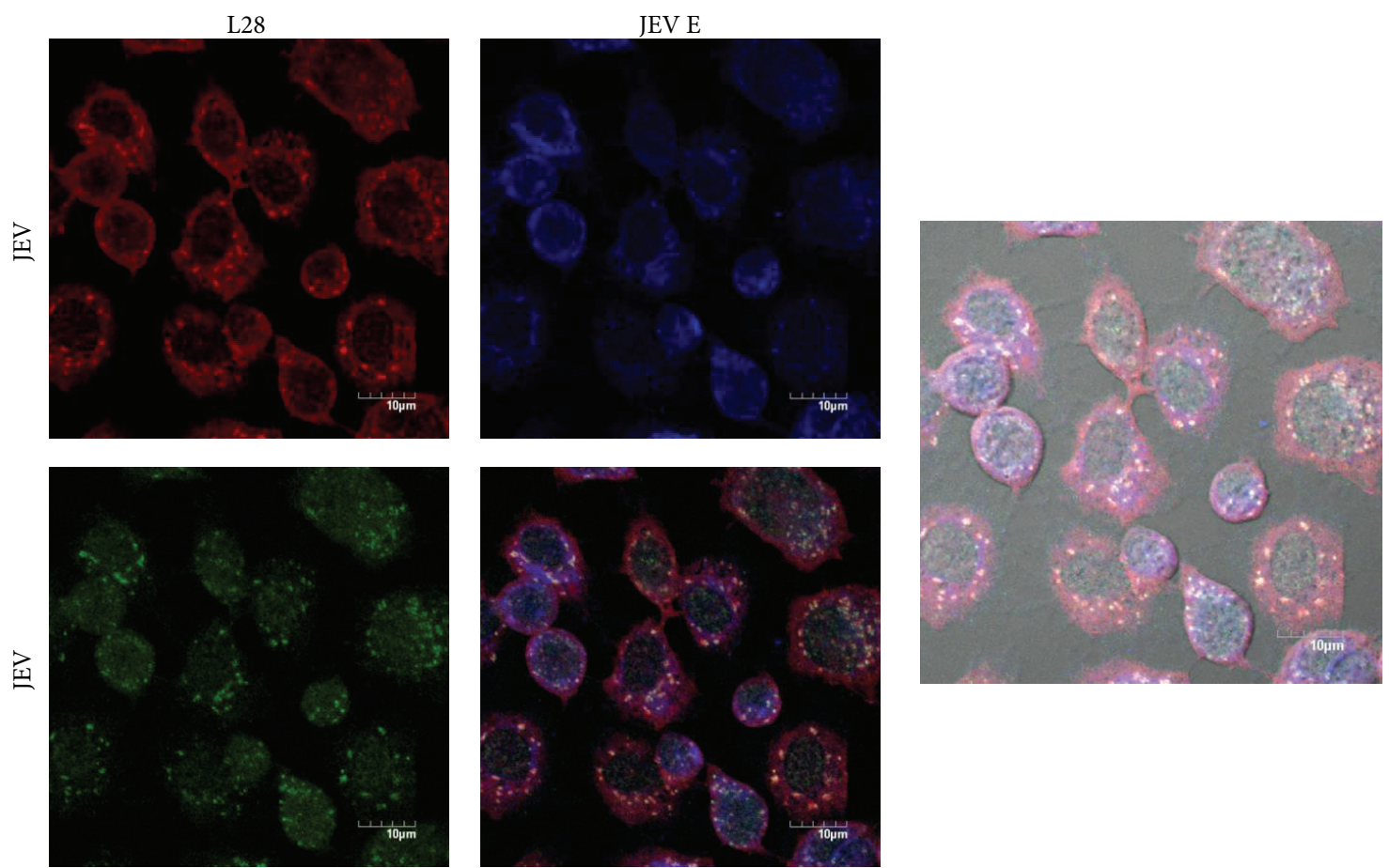

VDAC

Merge

FIGURE 5: Location of JEV E protein, VDAC, and ribosomal L28 protein in JEV infected C6/36 cells. C6/36 cells grown on glass cover slips were mock infected or infected with JEV and examined for location of JEV E protein (blue), ribosomal L28 protein (red), and VDAC (green) using an Olympus FluoView 1000 confocal microscope. Representative individual and merged images are shown, and one panel has bright field added. 

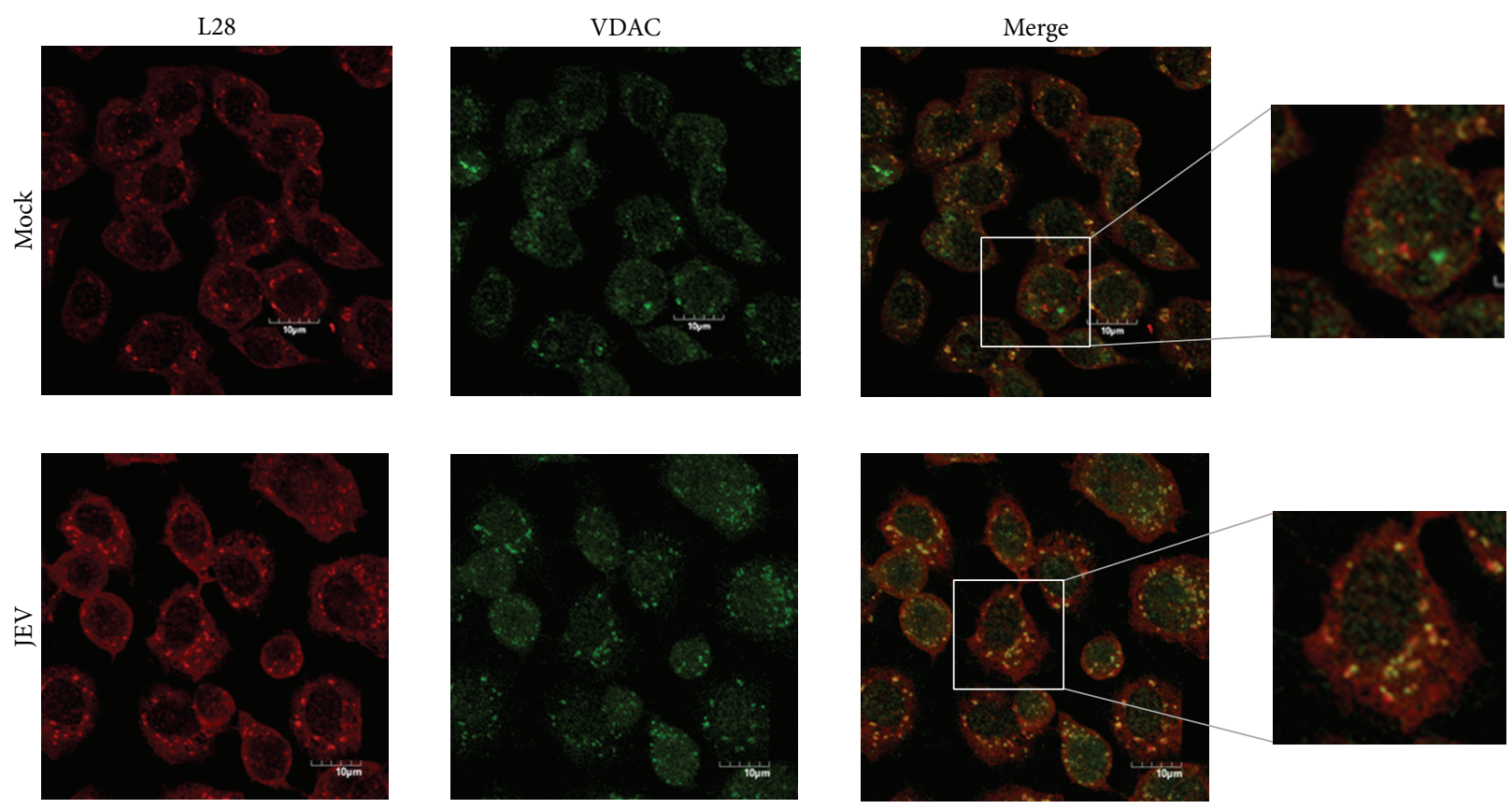

FIGURE 6: Location of JEV E protein, VDAC, and ribosomal L28 protein in JEV infected C6/36 cells. Signal of VDAC and ribosomal L28 protein extracted from Figure 5 for improved visualization purposes with enlargement.

important part of JEV replication in insect cells. Further study may elucidate whether such a mechanism similarly occurs in mammalian cells.

\section{Conflict of Interests}

The authors declare that there is no conflict of interests regarding the publication of this paper.

\section{Acknowledgments}

This work was supported by grants from the Office of the Higher Education Commission and Mahidol University under the National Research Universities Initiative and Mahidol University. Chanida Fongsaran is supported by a Thai Royal Golden Jubilee Ph.D. Scholarship.

\section{References}

[1] S. C. Weaver and A. D. T. Barrett, "Transmission cycles, host range, evolution and emergence of arboviral disease," Nature Reviews Microbiology, vol. 2, no. 10, pp. 789-801, 2004.

[2] S. Mukhopadhyay, R. J. Kuhn, and M. G. Rossmann, "A structural perspective of the Flavivirus life cycle," Nature Reviews Microbiology, vol. 3, no. 1, pp. 13-22, 2005.

[3] B. D. Lindenbach and C. M. Rice, "Molecular biology of flaviviruses," Advances in Virus Research, vol. 59, pp. 23-61, 2003.

[4] U. K. Misra and J. Kalita, "Overview: japanese encephalitis," Progress in Neurobiology, vol. 91, no. 2, pp. 108-120, 2010.

[5] S. C. Weaver and W. K. Reisen, "Present and future arboviral threats," Antiviral Research, vol. 85, no. 2, pp. 328-345, 2010.

[6] L. Rosen, "The natural history of Japanese encephalitis virus," Annual Review of Microbiology, vol. 40, pp. 395-414, 1986.
[7] A. F. van den Hurk, S. A. Ritchie, and J. S. Mackenzie, "Ecology and geographical expansion of japanese encephalitis virus," Annual Review of Entomology, vol. 54, pp. 17-35, 2009.

[8] J. S. Mackenzie, D. J. Gubler, and L. R. Petersen, "Emerging flaviviruses: the spread and resurgence of Japanese encephalitis, West Nile and dengue viruses," Nature Medicine, vol. 10, no. 12, pp. S98-S109, 2004.

[9] G. J. Sips, J. Wilschut, and J. M. Smit, "Neuroinvasive flavivirus infections," Reviews in Medical Virology, vol. 22, no. 2, pp. 69-87, 2012.

[10] T. Solomon, "Flavivirus encephalitis," The New England Journal of Medicine, vol. 351, no. 4, pp. 370-378, 2004.

[11] M. Kalia, R. Khasa, M. Sharma, M. Nain, and S. Vrati, "Japanese encephalitis virus infects neuronal cells through a clathrinindependent endocytic mechanism," Journal of Virology, vol. 87, no. 1, pp. 148-162, 2013.

[12] Y. Zhu, Q. Xu, D. Wu et al., "Japanese encephalitis virus enters rat neuroblastoma cells via a $\mathrm{pH}$ dependent, dynamin and caveola-mediated endocytosis pathway," Journal of Virology, vol. 86, no. 24, pp. 13407-13422, 2012.

[13] M. Nawa, T. Takasaki, K. Yamada, I. Kurane, and T. Akatsuka, "Interference in Japanese encephalitis virus infection of Vero cells by a cationic amphiphilic drug, chlorpromazine," Journal of General Virology, vol. 84, no. 7, pp. 1737-1741, 2003.

[14] S. Yang, M. He, X. Liu, X. Li, B. Fan, and S. Zhao, "Japanese encephalitis virus infects porcine kidney epithelial PK15 cells via clathrin- and cholesterol-dependent endocytosis," Virology Journal, vol. 10, article 258, 2013.

[15] S. Das, S. V. Laxminarayana, N. Chandra, V. Ravi, and A. Desai, "Heat shock protein 70 on Neuro2a cells is a putative receptor for Japanese encephalitis virus," Virology, vol. 385, no. 1, pp. 4757, 2009.

[16] Y. Z. Zhu, M. M. Cao, W. B. Wang, H. Ren, P. Zhao, and Z. Qi, "Association of heat-shock protein 70 with lipid rafts is required 
for japanese encephalitis virus infection in Huh7 cells," Journal of General Virology, vol. 93, no. 1, pp. 61-71, 2012.

[17] S. Das, V. Ravi, and A. Desai, "Japanese encephalitis virus interacts with vimentin to facilitate its entry into porcine kidney cell line," Virus Research, vol. 160, no. 1-2, pp. 404-408, 2011.

[18] Y. Chien, W. Chen, W. Hsu, and S. Chiou, "Bovine lactoferrin inhibits Japanese encephalitis virus by binding to heparan sulfate and receptor for low density lipoprotein," Virology, vol. 379, no. 1, pp. 143-151, 2008.

[19] T. Thongtan, N. Wikan, P. Wintachai et al., "Characterization of putative Japanese encephalitis virus receptor molecules on microglial cells," Journal of Medical Virology, vol. 84, no. 4, pp. 615-623, 2012.

[20] W. C. Black, K. E. Bennett, N. Gorrochótegui-Escalante et al., "Flavivirus susceptibility in Aedes aegypti," Archives of Medical Research, vol. 33, no. 4, pp. 379-388, 2002.

[21] M. Nawa, "Effects of bafilomycin A1 on Japanese encephalitis virus in C6/36 mosquito cells," Archives of Virology, vol. 143, no. 8, pp. 1555-1568, 1998.

[22] J. Ren, T. Ding, W. Zhang, J. Song, and W. Ma, "Does Japanese encephalitis virus share the same cellular receptor with other mosquito-borne flaviviruses on the C6/36 mosquito cells?" Virology Journal, vol. 4, article 83, 2007.

[23] V. Boonsanay and D. R. Smith, "Entry into and production of the Japanese encephalitis virus from C6/36 cells," Intervirology, vol. 50, no. 2, pp. 85-92, 2007.

[24] S. Jindadamrongwech, C. Thepparit, and D. R. Smith, "Identification of GRP 78 (BiP) as a liver cell expressed receptor element for dengue virus serotype 2," Archives of Virology, vol. 149, no. 5, pp. 915-927, 2004.

[25] T. Limjindaporn, W. Wongwiwat, S. Noisakran et al., "Interaction of dengue virus envelope protein with endoplasmic reticulum-resident chaperones facilitates dengue virus production," Biochemical and Biophysical Research Communications, vol. 379, no. 2, pp. 196-200, 2009.

[26] S. Upanan, A. Kuadkitkan, and D. R. Smith, "Identification of dengue virus binding proteins using affinity chromatography," Journal of Virological Methods, vol. 151, no. 2, pp. 325-328, 2008.

[27] D. T. Rutkowski and R. J. Kaufman, "A trip to the ER: coping with stress," Trends in Cell Biology, vol. 14, no. 1, pp. 20-28, 2004.

[28] M. Schröder, "Endoplasmic reticulum stress responses," Cellular and Molecular Life Sciences, vol. 65, no. 6, pp. 862-894, 2008.

[29] C. Xu, B. Bailly-Maitre, and J. C. Reed, "Endoplasmic reticulum stress: cell life and death decisions," Journal of Clinical Investigation, vol. 115, no. 10, pp. 2656-2664, 2005.

[30] A. Delpino and M. Castelli, "The $78 \mathrm{kDa}$ glucose-regulated protein (GRP78/BIP) is expressed on the cell membrane, is released into cell culture medium and is also present in human peripheral circulation," Bioscience Reports, vol. 22, no. 3-4, pp. 407-420, 2002.

[31] B. K. Shin, H. Wang, A. M. Yim et al., "Global profiling of the cell surface proteome of cancer cells uncovers an abundance of proteins with chaperone function," The Journal of Biological Chemistry, vol. 278, no. 9, pp. 7607-7616, 2003.

[32] M. Triantafilou, D. Fradelizi, and K. Triantafilou, "Major histocompatibility class one molecule associates with glucose regulated protein (GRP) 78 on the cell surface," Human Immunology, vol. 62, no. 8, pp. 764-770, 2001.

[33] G. Xiao, T. Chung, H. Y. Pyun, R. E. Fine, and R. J. Johnson, "KDEL proteins are found on the surface of NG108-15 cells," Molecular Brain Research, vol. 72, no. 2, pp. 121-128, 1999.
[34] M. A. Alhoot, S. M. Wang, and S. D. Sekaran, "RNA interference mediated inhibition of dengue virus multiplication and entry in HepG2 cells," PLoS ONE, vol. 7, no. 3, Article ID e34060, 2012.

[35] K. Triantafilou, D. Fradelizi, K. Wilson, and M. Triantafilou, "GRP78, a coreceptor for coxsackievirus A9, interacts with major histocompatibility complex class I molecules which mediate virus internalization," Journal of Virology, vol. 76, no. 2, pp. 633-643, 2002.

[36] T. Thongtan, P. Cheepsunthorn, V. Chaiworakul, C. Rattanarungsan, N. Wikan, and D. R. Smith, "Highly permissive infection of microglial cells by Japanese encephalitis virus: a possible role as a viral reservoir," Microbes and Infection, vol. 12, no. 1, pp. 37-45, 2010.

[37] M. M. Bradford, "A rapid and sensitive method for the quantitation of microgram quantities of protein utilizing the principle of protein-dye binding," Analytical Biochemistry, vol. 72, no. 1-2, pp. 248-254, 1976.

[38] S. Hung, P. Lee, H. Chen, L. Chen, C. Kao, and C. King, "Analysis of the steps involved in dengue virus entry into host cells," Virology, vol. 257, no. 1, pp. 156-167, 1999.

[39] M. Panyasrivanit, A. Khakpoor, N. Wikan, and D. R. Smith, "Co-localization of constituents of the dengue virus translation and replication machinery with amphisomes," Journal of General Virology, vol. 90, no. 2, pp. 448-456, 2009.

[40] M. D. Abramoff, P. J. Magelhaes, and S. J. Ram, "Image processing with image," Journal of Biophotonics International, vol. 11, pp. 36-42, 2004.

[41] A. P. French, S. Mills, R. Swarup, M. J. Bennett, and T. P. Pridmore, "Colocalization of fluorescent markers in confocal microscope images of plant cells," Nature Protocols, vol. 3, no. 4, pp. 619-628, 2008.

[42] M. Gonzalez-Gronow, S. J. Kaczowka, S. Payne, F. Wang, G. Gawdi, and S. V. Pizzo, "Plasminogen structural domains exhibit different functions when associated with cell surface GRP78 or the voltage-dependent anion channel," Journal of Biological Chemistry, vol. 282, no. 45, pp. 32811-32820, 2007.

[43] M. Gonzalez-Gronow, M. A. Selim, J. Papalas, and S. V. Pizzo, "GRP78: a multifunctional receptor on the cell surface," Antioxidants and Redox Signaling, vol. 11, no. 9, pp. 2299-2306, 2009.

[44] A. Nakatsuka, J. Wada, I. Iseda et al., "Visceral adipose tissue-derived serine proteinase inhibitor inhibits apoptosis of endothelial cells as a ligand for the cell-surface GRP78/voltagedependent anion channel complex," Circulation Research, vol. 112, no. 5, pp. 771-780, 2013.

[45] M. Ni, Y. Zhang, and A. S. Lee, "Beyond the endoplasmic reticulum: atypical GRP78 in cell viability, signalling and therapeutic targeting," Biochemical Journal, vol. 434, no. 2, pp. 181-188, 2011.

[46] M. Colombini, "VDAC structure, selectivity, and dynamics," Biochimica et Biophysica Acta: Biomembranes, vol. 1818, no. 6, pp. 1457-1465, 2012.

[47] V. Shoshan-Barmatz and M. Golan, "Mitochondrial VDAC1: function in cell life and death and a target for cancer therapy," Current Medicinal Chemistry, vol. 19, no. 5, pp. 714-735, 2012.

[48] Y. Shi, J. Chen, C. Weng et al., "Identification of the protein-protein contact site and interaction mode of human VDAC1 with Bcl-2 family proteins," Biochemical and Biophysical Research Communications, vol. 305, no. 4, pp. 989-996, 2003.

[49] V. Shoshan-Barmatz and A. Israelson, "The voltage-dependent anion channel in endoplasmic/sarcoplasmic reticulum: Characterization, modulation and possible function," Journal of Membrane Biology, vol. 204, no. 2, pp. 57-66, 2005. 
[50] V. Shoshan-Barmatz, R. Zalk, D. Gincel, and N. Vardi, "Subcellular localization of VDAC in mitochondria and ER in the cerebellum," Biochimica et Biophysica Acta-Bioenergetics, vol. 1657, no. 2-3, pp. 105-114, 2004.

[51] L. G. Gebhard, S. B. Kaufman, and A. V. Gamarnik, "Novel ATPindependent RNA annealing activity of the dengue virus NS3 helicase," PLoS ONE, vol. 7, no. 4, Article ID e36244, 2012. 

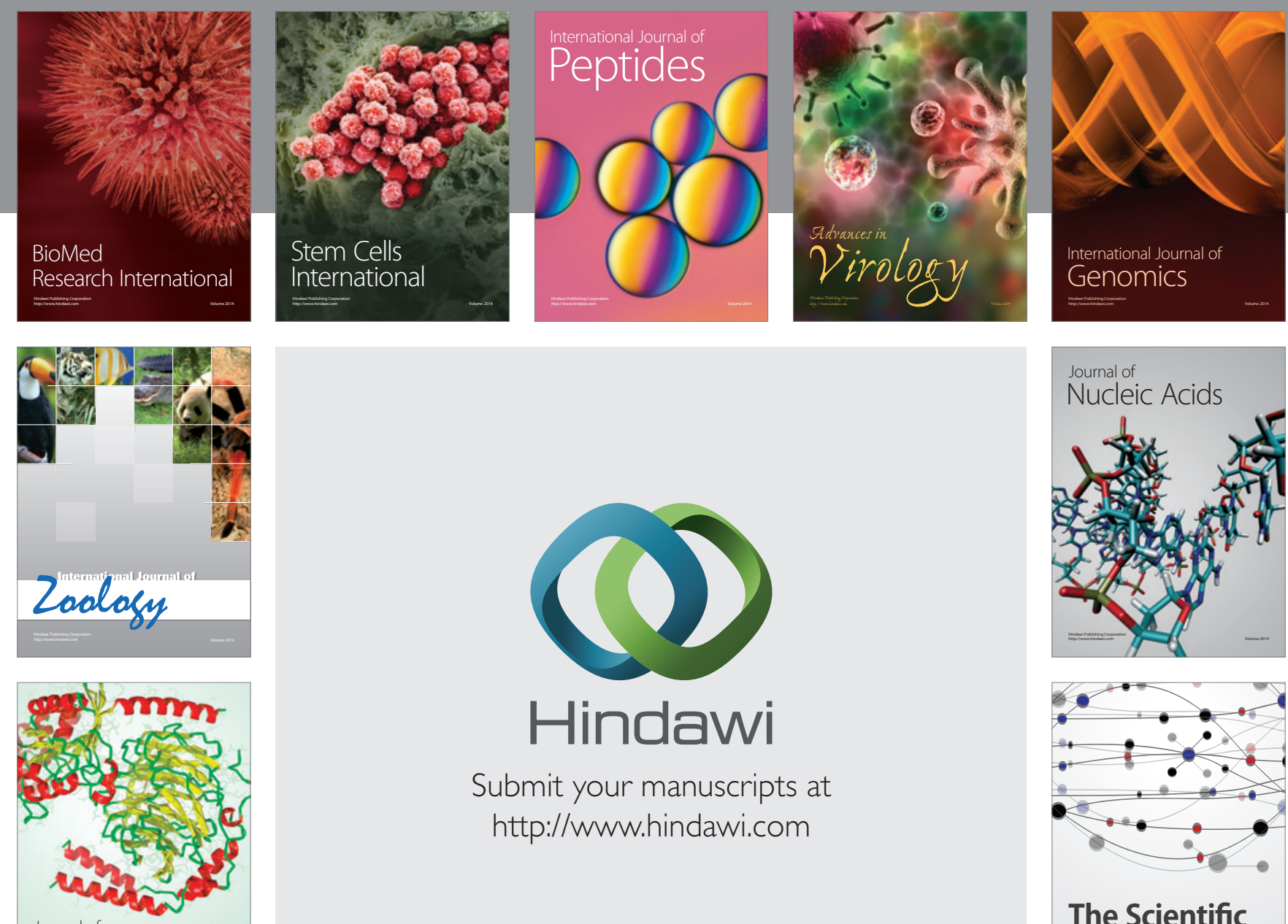

Submit your manuscripts at

http://www.hindawi.com

Journal of
Signal Transduction
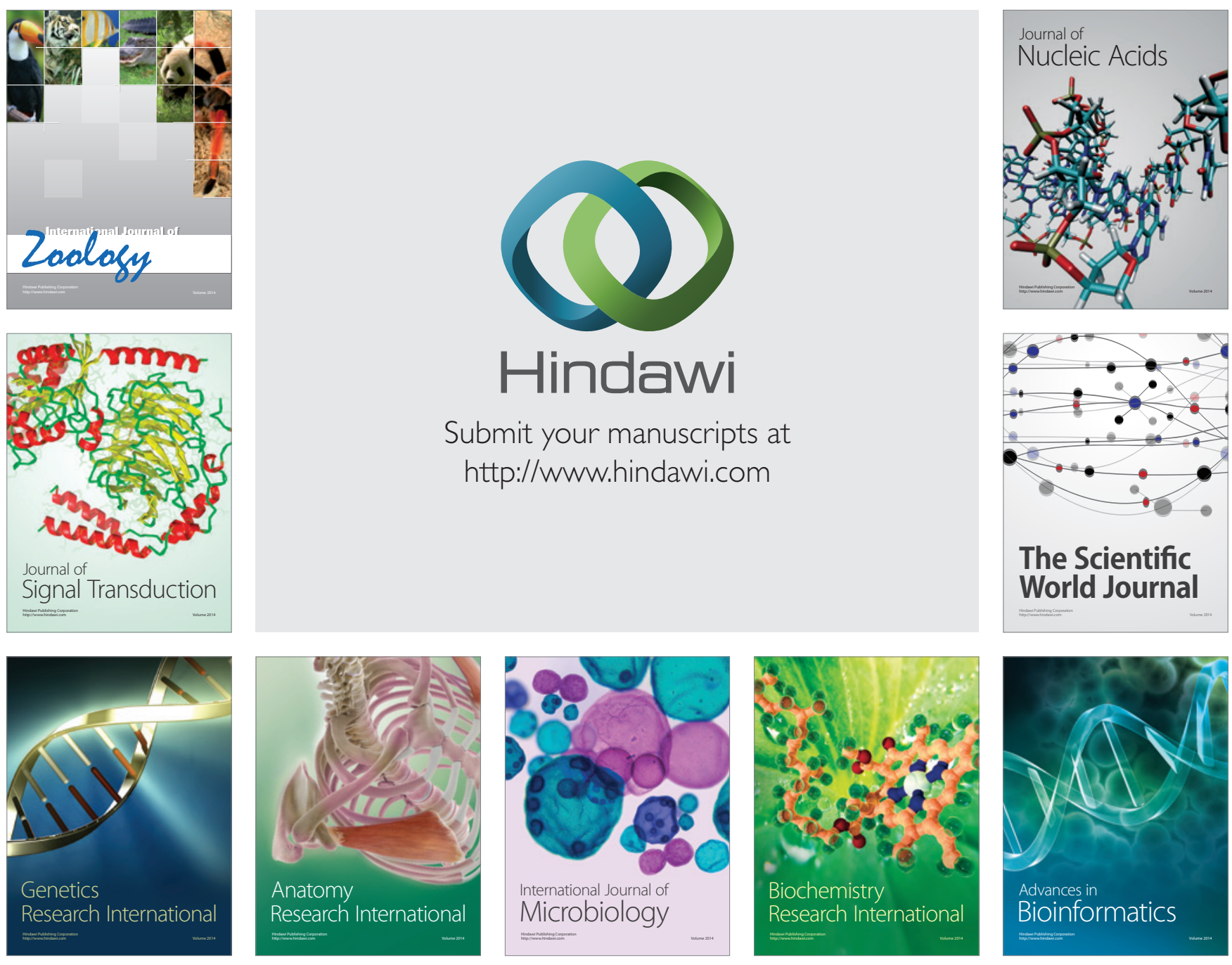

The Scientific World Journal
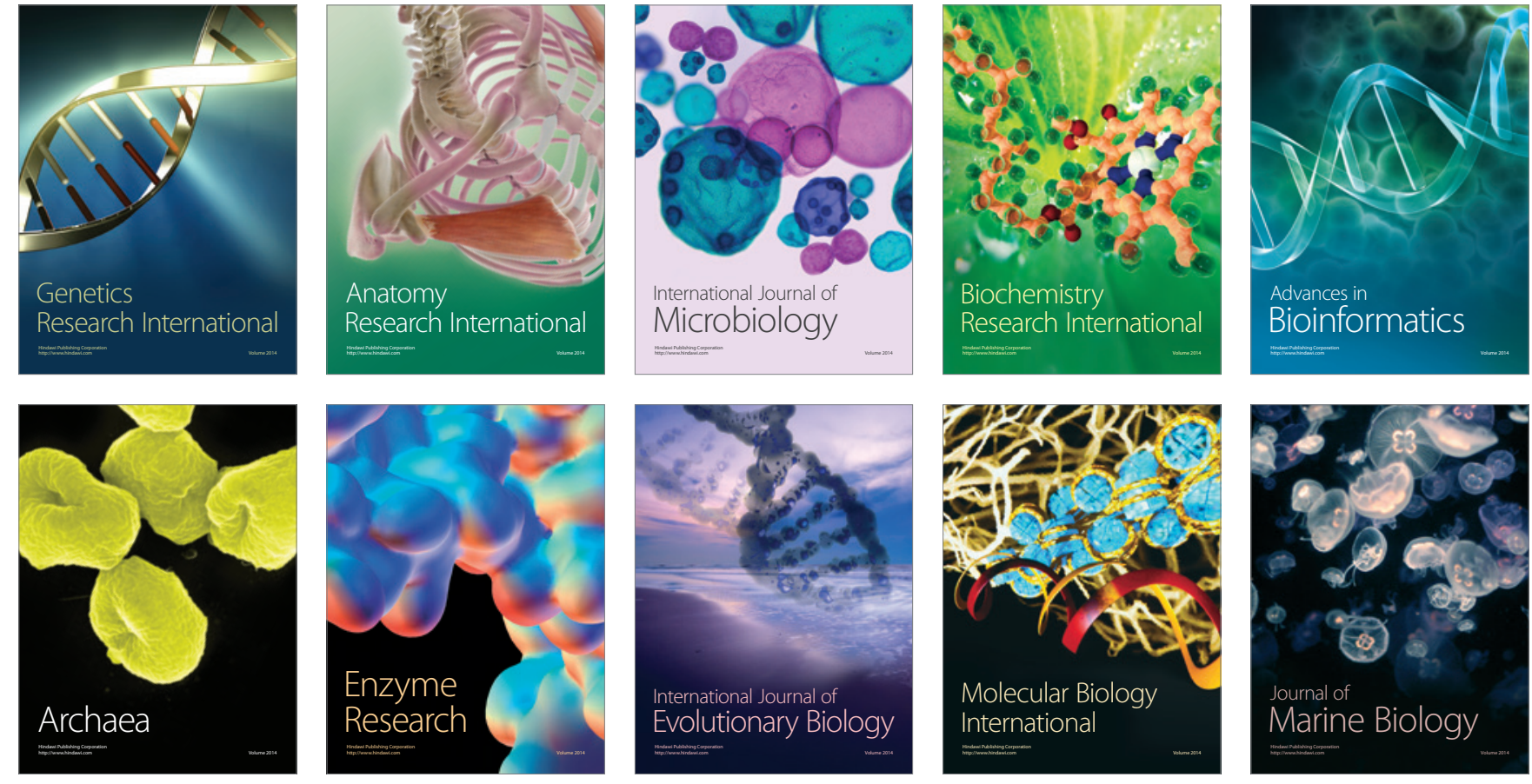